\title{
Metodología para la evaluación integral de un proyecto de modernización de instrumentación
}

\author{
L.R. Vega-González \\ Centro de Instrumentos, UNAM \\ Unidad de Inteligencia Tecnológica y Departamento de Ingeniería y Servicios \\ E-mail:1veg@pumas.iingen.unam.mx
}

(recibido: noviembre de 1998; aceptado: febrero de 2001)

\begin{abstract}
Resumen
El presente trabajo es el resultado de un estudio presentado a la Dirección General de Construcción y Operación Hidráulica (DGCOH) del Departamento del Distrito Federal ${ }^{1}$ sobre la evaluación de la factibilidad de modernizar la instrumentación y control de la Planta de Tratamiento de Aguas Residuales (PTAR) Chapultepec. A diferencia de proyectos de inversión nuevos, la evaluación de un proyecto de modernización presenta algunos aspectos interesantes, ya que se trata de plantas que cuentan con muchos años de operación, lo que restringe los posibles beneficios del proyecto. De la misma forma, la vida útil de la planta prácticamente esta agotada. A lo anterior, se agrega el hecho de que en una dependencia gubernamental que opera prácticamente basándose en subsidios, los beneficios muchas veces son intangibles, ya que la operación de las plantas no tiene una finalidad de lucro, sino de servicio a la comunidad. Por si esto fuera poco, al no existir una conciencia ecológica sobre la importancia que tienen las Plantas de Tratamiento de Aguas Residuales para evitar mayores impactos ambientales. Con este panorama, no resultan obvios los factores de beneficio en la mente de los tomadores de decisión. A continuación se presenta una propuesta metodológica para la evaluación integral de este tipo de proyectos de modernización.
\end{abstract}

Descriptores: evaluación de proyectos, proyectos de modernización, organizaciones de servicio gubernamentales, instrumentación industrial, tratamiento de aguas.

\section{Abstract}

This work is the result of a study financed by the Mexico City government through its DGCOH regarding the feasibility of retrofitting the instrumentation and control for the Chapultepec Waste Water Treatment Plant. As opposed to new investment projects, the evaluation of a retrofit project presents some interesting aspects since in this case the plants typically have been operating for many years restricting the possible benefits since the plant life cycle has practically expired. In addition, it is a known fact that a governmental operating organization works on a subsidized basis, making benefits intangible since it is not a profitable operation but rather a community service. To make things worse, there is not an ecological awareness in Mexico about the importance of municipal wastewater treatment plants to prevent major environmental impacts. In this scheme, the beneficial factors are not so obvious to decision makers. A methodological proposal is presented for the integral evaluation of this kind of retrofit projects.

Keywords: project evaluation, retrofit projects, service organizations, industrial instrumentation, water treatment.

\section{Antecedentes}

La Planta de Tratamiento de Aguas Residuales de Chapultepec de la DGCOH del GDF, fue diseñada para tratar un caudal de 180 litros por segundo de aguas residuales residenciales, conocidas como municipales. Se trata de una

\footnotetext{
1 Actualmente Gobierno del Distrito Federal (GDF)

la DGCOH Direccion de Construcción y Operación Hidráulica del Departamento del Distrito Federal.
} 
planta de tratamiento biológico secundario que opera en dos unidades, en las cuales resalta la configuración geométrica de los tanques de sedimentación primaria, ya que en la unidad No. 1 el tanque es cilíndrico, mientras que en el tanque No.2 es de base rectangular. La planta fue puesta en operación en 1956, por lo que cuenta ya con 45 años de operación continua.

El equipo de instrumentación instalado originalmente fue muy escaso, y actualmente sólo existen unos cuantos instrumentos de monitoreo para variables de análisis y para registro de los caudales. La inspección preliminar indicó que la calibración de los instrumentos era muy dudosa, en el caso de los sistemas de monitoreo y registro de caudal se trata de instrumentación que ya está obsoleta, por lo que ya no es posible encontrar refacciones en el mercado.

Ante esta situación, en el año de 1998 se planteó la posibilidad de realizar un proyecto de modernización de la instrumentación para reemplazar los equipos inoperantes, así como implementar un sistema de control para mejorar la operación integral de la planta (management), permitiendo su enlace con otras estaciones de supervisión exteriores, mejorando así, la eficiencia de operación de la planta.

Este planteamiento hizo necesaria la ejecución de un sub-proyecto de ingeniería básica para la instrumentación y control, con un levantamiento exhaustivo de las condiciones de la planta y la exposición de nuevas condiciones de diseño. Como resultado de los trabajos de ingeniería, se emitieron nuevos diagramas de tubería e instrumentación, posteriormente se hizo una propuesta detallada de los nuevos instrumentos requeridos, de acuerdo con los criterios establecidos por los ingenieros de proceso.

\section{Efecto de un proyecto de modernización en una unidad productiva}

Para este análisis se considerará a la planta de tratamiento de aguas residuales como unidad productiva, cuyo producto final es agua tratada de acuerdo a ciertas especificaciones y normas ambientales.

El desarrollo de la metodología, parte de la identificación de los efectos que un proyecto de modernización generará en una unidad que se encuentra en plena operación.

Para esto utilizamos el concepto de vectores de cambio, según las siguientes definiciones:

VCEI $=$ Vector de Cambio en los Efectos Internos de la unidad productiva.

VCEE $=$ Vector de Cambio en los Efectos Externos

Estos conceptos se muestran gráficamente en las figuras 1 y 2. Al someter la unidad productiva a un proyecto de modernización, se experimentarán cambios en la magnitud y tipo de "efectos externos" que son producidos en la sociedad, la comunidad y el medio ambiente que le circunda.

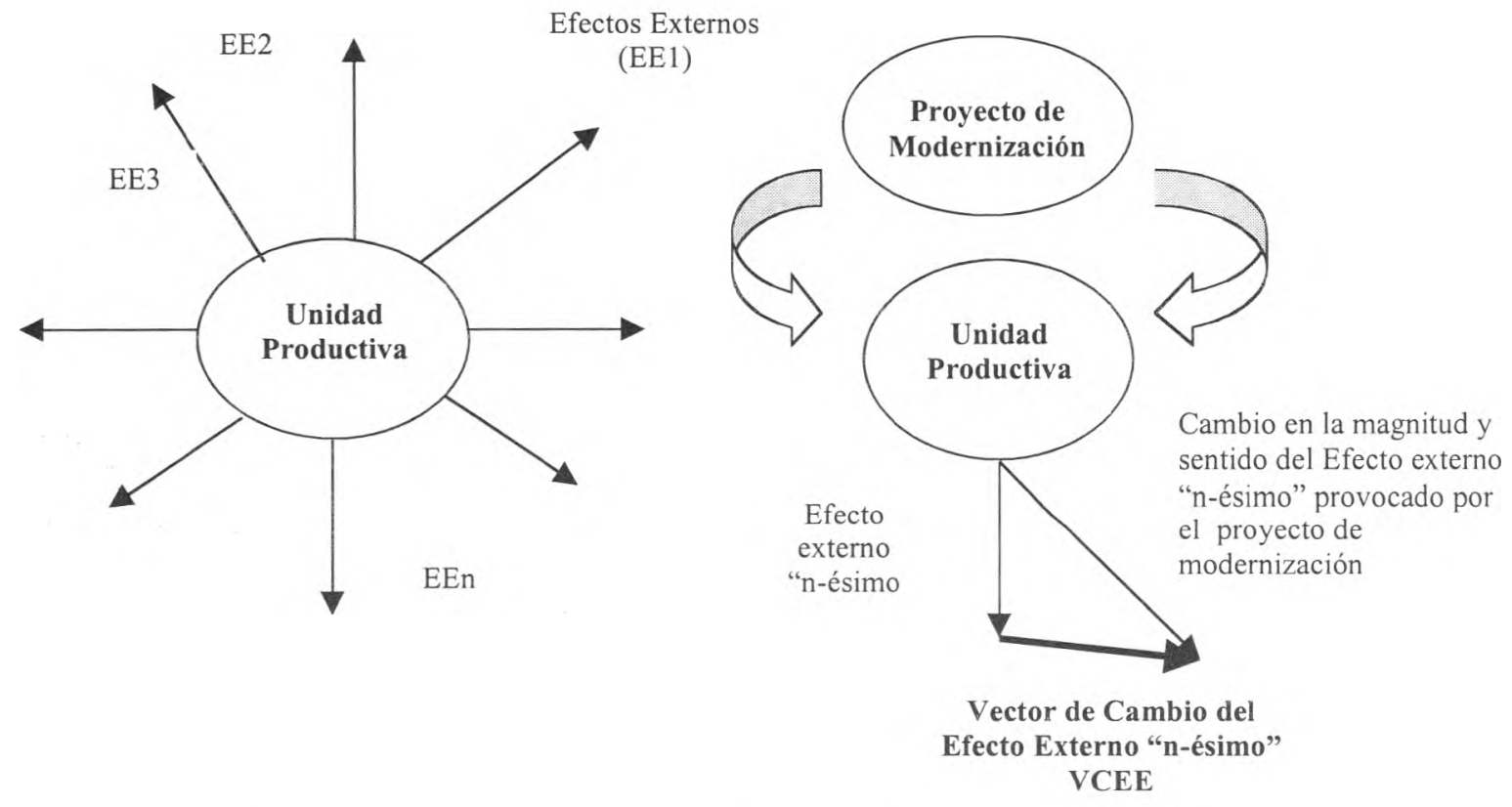

Figura 1. Concepto de Vector de Cambio de Efectos Externos (VCEE)

Similarmente con la ejecución del proyecto de modernización, se experimentan cambios de cierta magnitud y sentido al interior de la unidad productiva. A estos los hemos denominado como "efectos internos". 


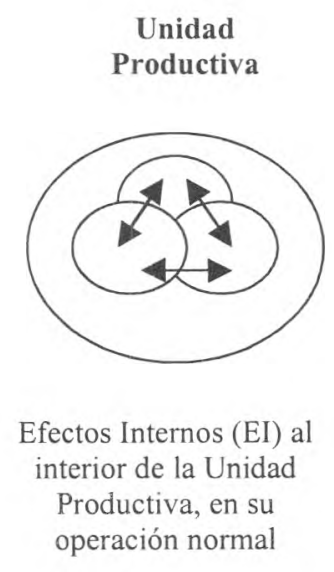

Unidad Productiva

Efectos Internos (EI) al interior de la Unidad operación normal

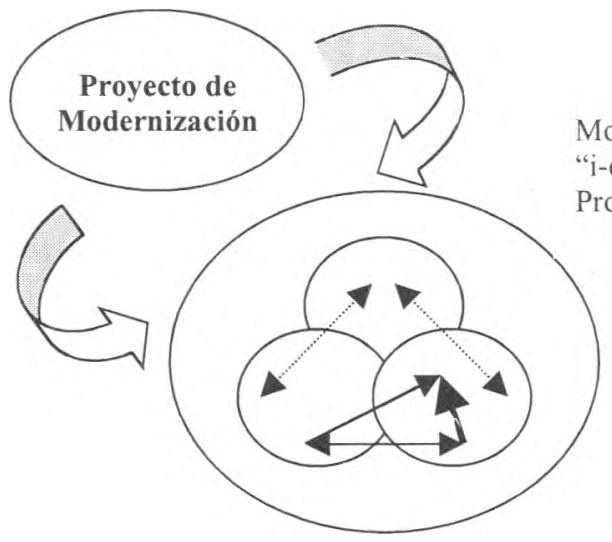

Modificación del Efecto Interno "i-ésimo" al presentarse el

Proyecto de Modernización

\section{Vector de Cambio \\ en el efecto Interno \\ "i-ésimo" (VCEI)}

Efecto Interno "i-ésimo"

antes del proyecto de

modernización

\section{Figura 2. Concepto de Vector de Cambio de Efectos Internos (VCEI)}

Así es como los elementos que ligan la situación en cuanto a efectos internos y externos originales en la unidad productiva y los cambios que se dan al ejecutarse el proyecto de modernización, son los vectores de cambio VCEE y VCEI.

\section{Metodología propuesta para la evaluación de la factibilidad del proyecto de modernización}

Contando con los conceptos de Vector de Cambio de Efectos Internos y Externos, estamos en la posibilidad de proponer un método de evaluación integral para demostrar la factibilidad ${ }^{2}$ del proyecto de modernización.

El primer paso consiste en el uso de herramientas para identificar la magnitud, principalmente, el sentido de los vectores de cambio, ya que resulta natural que un cambio negativo en los efectos externos o internos, afectará la aceptación del proyecto, y esta descalificación se acentuará entre mayor sea la magnitud del cambio negativo. Por el contrario, cambios positivos en los efectos externos e internos, marcarán la aceptación del proyecto de modernización, la cual solamente quedará sujeta a la valoración económica-financiera tradicional, sea por cualquier método de análisis financiero que se utilize.

De acuerdo con Soumelis (1977), evaluación es la función por medio de la cual se obtienen resultados actuales o eventuales de un curso de acción específico,

${ }^{2}$ Se habla de la evaluación de la factibilidad del proyecto de modernización, porque el ejercicio se realiza antes de la aceptación y ejecución del proyecto; sin embargo, el método propuesto también puede utilizarse para la evaluación de los proyectos ya ejecutados en un análisis "ex-post". calificándolo contra un criterio de evaluación. Este último es un juego independiente de alternativas establecidas en parámetros, los cuales proveen medidas directas de los resultados de los cursos de acción alternativos. Para nues. tros fines, la tabla 1 muestra los criterios de aceptación o rechazo de proyectos, usando en la primera y segunda columnas el sentido de los vectores de cambio externos e internos y en la tercera columna la Valoración Financiera Costo/Beneficio (VFC/B) o la valoración financiera por Valor Presente Neto (VPN).

Para el primer caso, se consideran positivas las razones $C / B>1$ y negativos los proyectos cuyo índice sea $\mathrm{C} / \mathrm{B}<1$. Para el segundo, se consideran positivos los valores del VPN>1 y negativos en caso contrario. Cuando el índice C/B es igual a uno no hay decisión para la aceptación del proyecto. Lo mismo sucede cuando el VPN es igual a cero.

Los métodos tradicionales para determinar la factibilidad de un proyecto, se basan exclusivamente en la realización de un ejercicio de evaluación económica y financiera del proyecto, en términos de los costos asociados con el mismo y los posibles beneficios globales y particulares de la propuesta.

Algunos autores como Ranashinge (1997), han discutido sobre la necesidad de reconciliar los elementos de la utilidad privada con los costos sociales. Otros como Hinterhuber (1997), apoyan esta noción, ya que nos indican que hoy en día no es posible hablar de los "costos de un proceso", o de una mejora al mismo en forma aislada, sino que es "necesario ver el todo antes que las partes" (Scharnhorst). Nardini (1997), amplió el análisis 
utilizando una evaluación multicriterio, tomando como elementos en el proceso de toma de decisiones (DM), la evaluación de tres categorías básicas: el análisis de impacto ambiental (EIA), el análisis costo-beneficio (CBA), y el análisis multicriterio (MCA), incluyendo en este último la solución de conflictos interorganizacionales.

Para la evaluación de este proyecto, el criterio utilizado fue el de reforzar el método de evaluación con el "enfoque sistémico", para abordar el problema de una forma más integral que involucrara tanto las variables de costos y productividad, como la de impactos sociales y tecnológicos al interior y exterior de la unidad productiva.
La diferencia en el enfoque, radica en que no se trata sólo de realizar el análisis de los factores económico-financieros, sino de considerar también la forma en que el proyecto influirá en la vida de los propios operadores de las plantas, de la sociedad en su conjunto, e incluso, del impacto ecológico esperado con el nuevo proceso.

Por ello es que para este método de evaluación, los vectores de cambio de los efectos externos, se miden considerando los impactos a la sociedad, a la comunidad y al ecosistema que rodea a la planta, mientras que los vectores de cambio de los efectos internos se miden analizando los efectos sobre aspectos estratégicos que el proyecto de modernización traerá al interior de la planta.

Tabla 1. Criterios de aceptación o rechazo de proyectos

\begin{tabular}{|c|c|c|c|c|}
\hline VCEE & VCEI & VF C/B VPN & Aceptación o rechazo del proyecto & Notas \\
\hline 0 & 0 & 0 & $* * *$ & Sin elementos de decisión \\
\hline 0 & 0 & - & Rechazo & \\
\hline 0 & 0 & + & Aceptación condicionada & $\begin{array}{l}\text { Un proyecto de modernización debe provocar cambios, verificar } \\
\text { análisis }\end{array}$ \\
\hline - & 0 & 0 & Rechazo & \\
\hline- & 0 & - & Rechazo & \\
\hline- & 0 & + & Aceptación condicionada & $\begin{array}{l}\text { Verificar la magnitud de los cambios externos negativos, producto } \\
\text { de P. de modernización }\end{array}$ \\
\hline+ & 0 & 0 & $* * *$ & Sin elementos suficientes de decisión \\
\hline+ & 0 & - & Rechazo & $\begin{array}{l}\text { La aceptación de estos casos puede darse por situaciones políticas, } \\
\text { dependiendo de la magnitud y tipo de los VCEE involucrados }\end{array}$ \\
\hline+ & 0 & + & Aceptación & \\
\hline 0 & - & - & Rechazo & \\
\hline 0 & - & 0 & Rechazo & \\
\hline 0 & - & + & Aceptación condicionada & $\begin{array}{l}\text { Verificar la magnitud del vector de cambios internos, así como la } \\
\text { magnitud del índice de valoración financiera }\end{array}$ \\
\hline- & - & - & Rechazo & \\
\hline- & - & 0 & Rechazo & \\
\hline- & - & + & Rechazo & $\begin{array}{l}\text { Este tipo de proyectos se logra aceptar, si la magnitud del índice de } \\
\text { evaluación financiera es muy grande, aunque el costo de su } \\
\text { ejecución será en los efectos sociales, ecologicos al interior de la } \\
\text { cultura organizacional }\end{array}$ \\
\hline+ & - & - & Rechazo & \\
\hline+ & - & 0 & Rechazo & \\
\hline+ & - & + & Aceptación condicionada & Verificar la magnitud y tipo de cambios negativos internos \\
\hline 0 & + & - & Rechazo & \\
\hline 0 & + & 0 & $* * *$ & Sin elementos suficientes para decidir \\
\hline 0 & + & + & Aceptación & \\
\hline- & + & - & Rechazo & \\
\hline- & + & 0 & Rechazo & \\
\hline- & + & + & Aceptación condicionada & $\begin{array}{l}\text { Verificar magnitud y tipo de cambios negativos en los efectos } \\
\text { externos }\end{array}$ \\
\hline+ & + & - & Rechazo & Este tipo de proyectos se llega a aceptar por razones políticas \\
\hline+ & + & 0 & $* * *$ & $\begin{array}{l}\text { Sin elementos suficientes para aceptar, este tipo de proyectos se } \\
\text { acepta por razones políticas }\end{array}$ \\
\hline+ & + & + & Aceptación absoluta & \\
\hline
\end{tabular}




\section{Definición de criterios básicos para la evaluación}

Para que exista congruencia en la valoración de los vectores de cambio VCEE, VCEI y la valoración económico-financiera del proyecto, se deben adoptar y establecer claramente los criterios de trabajo básicos. En este caso, los criterios fueron:

* Aunque el Gobierno del DF y la DGCOH son organizaciones públicas no lucrativas, es conveniente adoptar una visión empresarial, ya que esto permite visualizar el panorama del estado actual y futuro, al cual desea dirigirse la organización para orientar todas las acciones estratégicas tomadas.

* Las políticas y reglas de la misma organización, serán las que definirán las reglas del juego en el proyecto, es decir, el proyecto se ciñe a las reglas de la organización.

* Deberán definirse los criterios de satisfacción para todos los actores relacionados directa o indirectamente con el proyecto.

* Las plantas de tratamiento de aguas residuales de la DGCOH, pueden entenderse como unidades estratégicas de negocios o centros de acción colectiva que deben estar integrados y coordinados por la estrategia global de la organización.

* Al entender una planta de tratamiento de agua residual como una unidad estratégica, motiva al personal y lo impulsa a enrolarse en un proceso de aprendizaje permanente, evitando así reacciones de oposición al cambio y promoviendo la rápida asimilación de los nuevos sistemas y tecnologías.

\section{Desarrollo de la herramienta de medición para el VCEE}

La herramienta para medir el VCEE, realizará la evaluación del proyecto en sus impactos y servicio aportado a la sociedad, a la comunidad y a la ecología de una manera integral. Para ello, hay que considerar la participación de los directivos responsables de las áreas funcionales, de los responsables de las unidades de proceso o, de la planta en su conjunto; entre ellos, operadores, usuarios del producto del proceso, la comunidad en general, ya que todos y cada uno de los actores mencionados están relacionados con la operación de las plantas de tratamiento de agua residual y la ejecución de un proyecto de modernización, el cual modificará de una o de otra forma las condiciones de vida y operación de la planta y de ellos mismos.

Por lo tanto, los conceptos y criterios económicos aislados de costo de equipamiento y proyecto ejecutivo quedan rebasados como elementos únicos de evaluación y son medidos no sólo contra los atributos de beneficio, sino contra los de satisfacción de los diferentes actores involucrados.

Sobre la base de la visión anterior, se propone que al tratarse de un proyecto de modernización, sea indispensable mostrar en primera instancia, la forma de cómo los cambios propuestos afectan en términos de atributos de satisfacción a toda la comunidad y personal que estarían relacionados directa o indirectamente con el proyecto.

En el caso de la evaluación integral de proyectos múlti. ples, en un contexto de desarrollo integral, existen algunas herramientas desarrolladas para realizar la evaluación social (Sciara,1996), como son: matrices que incluyen coeficientes de productividad, de distribución del ingreso, impacto regional, impacto sobre la balanza de pagos, de impacto sobre el medio ambiente y sobre la organización social.

Para este proyecto utilizamos la herramienta de medi. ción adaptada y diseñada para la evaluación del VCEE presentada en la tabla 2, llamada Matriz de Valoración del Vector de Cambios en los Efectos Externos.

En la primera columna se hizo un listado exhaustivo de los distintos actores relacionados con el proyecto, mientras que en la segunda se incluyeron los Atributos de Satisfacción (AS) que ofrecía el proyecto a los actores relacionados. Los AS se ponderaron y calificaron por medio de un sistema de escala con cero al centro, de acuerdo con el peso relativo que tienen entre los diferentes actores involucrados.

Se asignó una calificación para cada factor en términos de una comparación subjetiva, la cual sería el estado de la variable en el escenario donde el proyecto fuese ejecutado. En la última columna se muestran algunas recomendaciones sobre acciones de mejora ${ }^{3}$.

\section{Desarrollo de la herramienta de medición para el VCEI}

Para medir el VCEI, se requierió el uso de una herramienta para medir los cambios que traería el proyecto de modernización en algunos aspectos estratégicos de la organización.

Para estar en condiciones de medir este efecto, se propuso construir el mapa de tendencias posibles de las mejoras estratégicas que podrían derivarse del proyecto.

Con este fin, adaptamos el concepto de Modelo de cambio de Cardiff para procesos de negocios, citado por Towill (1997).

\footnotetext{
${ }^{3}$ El ejercicio de aplicación del método a este caso de estudio, contiene calificaciones un tanto subjetivas por parte de quien realizó la evaluación, por lo tanto, se sugiere que la calificación de los atributos de satisfacción y de efectos externos sea realizada en forma participativa con personal técnico y operativo de la propia dependencia, quienes ejecutarán el proyecto en caso de ser aceptado.
} 
En este modelo se reconoce que en el proceso de cambio de la unidad de negocios, se emplean las tres componentes de mayor importancia, las cuales son comparables entre sí. El factor de actitud (actitudinal), el organizacional y el tecnológico (Figura 3).

Evidentemente, para el caso de este estudio el proceso de cambio lo representa el proyecto de modernización.

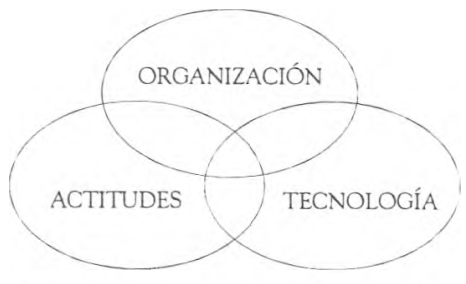

Figura 3. Modelo de cambio de Cardiff para procesos de negocios

Tabla 2. Matriz de valoración del Vector de Cambios en los Efectos Externos (VCEE)

\begin{tabular}{|c|c|c|c|c|c|c|c|c|c|}
\hline $\begin{array}{c}\text { Actor } \\
\text { involucrado }\end{array}$ & Atributo de Satisfacción & Peso & $\begin{array}{c}\text { Peor } \\
-2\end{array}$ & -1 & 0 & +1 & $\begin{array}{c}\text { Mejor } \\
+2 \\
\end{array}$ & Puntuación & Acciones de mejora \\
\hline $\begin{array}{l}\text { Clientes: } \\
\text { usuarios }\end{array}$ & $\begin{array}{c}\text { Precio } \\
\text { Calidad del Servicio } \\
\text { Calidad del agua }\end{array}$ & $\begin{array}{l}10 \\
10 \\
10\end{array}$ & & 晓 & 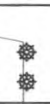 & & & $\begin{array}{c}-10 \\
0 \\
0\end{array}$ & $\begin{array}{l}\text { Venta de agua tratada a } \\
\text { particulares }\end{array}$ \\
\hline $\begin{array}{l}\text { Empleados } \\
\text { Directivos }\end{array}$ & $\begin{array}{c}\text { Estabilidad en el trabajo } \\
\text { Ambiente de trabajo } \\
\text { Calidad de la gerencia } \\
\text { Mejora del control de la planta } \\
\text { Intercambio de información } \\
\text { Cálculos de consumos energéticos y } \\
\text { reactivos } \\
\text { Registro histórico de operación } \\
\text { Actualización tecnológica }\end{array}$ & $\begin{array}{c}10 \\
8 \\
7 \\
7 \\
6 \\
7 \\
8 \\
8\end{array}$ & & & 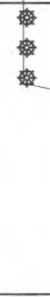 & 㑊 & & $\begin{array}{c}0 \\
0 \\
0 \\
7 \\
12 \\
7 \\
8 \\
16\end{array}$ & $\begin{array}{l}\text { Opciones de mejora para el } \\
\text { personal con el entrenamiento } \\
\text { respectivo. } \\
\text { Diseño del programa de } \\
\text { consumo energético } \\
\text { Uso del registro histórico de } \\
\text { operación para plan de } \\
\text { mantenimiento }\end{array}$ \\
\hline $\begin{array}{l}\text { Comunidad } \\
\text { en general }\end{array}$ & $\begin{array}{c}\text { Protección Ambiental } \\
\text { Creación y Mantenimiento de empleos } \\
\text { Disponibilidad de agua para otros fines } \\
\text { Diversión }\end{array}$ & $\begin{array}{l}10 \\
10 \\
8 \\
9\end{array}$ & & & 政 & 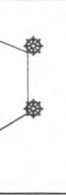 & & $\begin{array}{c}0 \\
10 \\
8 \\
0\end{array}$ & $\begin{array}{l}\text { Creación de criaderos de } \\
\text { trucha para pesca, cobrando } \\
\text { según tamaño de } \\
\text { la pieza, posibilidad de usarlas } \\
\text { como alimento }\end{array}$ \\
\hline Proveedores & $\begin{array}{c}\text { Nuevo mercado de refacciones } \\
\text { Nuevo Mercado de contratistas de } \\
\text { obra }\end{array}$ & $\begin{array}{l}10 \\
10\end{array}$ & & & & 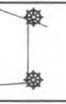 & & $\begin{array}{l}10 \\
10\end{array}$ & $\begin{array}{l}\text { Establecer programa de } \\
\text { desarrollo para garantizar } \\
\text { refacción. }\end{array}$ \\
\hline $\begin{array}{c}\text { Gobierno del } \\
\text { Distrito Federal }\end{array}$ & $\begin{array}{c}\text { Retorno de la inversión } \\
\text { Tiempo de uso (vida útil) } \\
\text { Atención de comunidad Polanco- } \\
\text { Chapultepec }\end{array}$ & $\begin{array}{c}6 \\
8 \\
10\end{array}$ & 嗦 & & & & & $\begin{array}{c}-12 \\
-6 \\
20\end{array}$ & $\begin{array}{c}\text { Reducción del ROI cobrando } \\
\text { el agua producida y } \\
\text { aumentando la eficiencia }\end{array}$ \\
\hline $\begin{array}{c}\text { Otras } \\
\text { Organizaciones } \\
\text { Plantas o } \\
\text { sistemas }\end{array}$ & $\begin{array}{c}\text { Interconectabilidad con nodo central } \\
\text { Interconectabilidad con sistemas } \\
\text { superiores }\end{array}$ & 7 & & & & & & $\begin{array}{l}7 \\
0\end{array}$ & Proyecto ejecutivo posterior \\
\hline
\end{tabular}

Para la elaboración de la herramienta de medición matricial, hubo que considerar a los factores operacionales de entrada y salida como los renglones de la matriz. Los factores operacionales de entrada a la unidad de negocios, fueron considerados como aquellas modificaciones que traería el proyecto a la planta, mientras que los factores operacionales de salida fueron entendidos como la estimación del beneficio del negocio individual, o de la planta, así como el sistema de beneficios que se generarán en cadena por el proyecto.

En las columnas se establecieron las estrategias de mejora consideradas como aquellos conceptos de mayor importancia, relativos a la optimización en el flujo de materiales e información en la planta, que surgirían en caso de ejecución del proyecto, y se medirían contra los factores operacionales en forma cruzada. También fue necesario incluir cualquier otro concepto de importancia mayor que pudiera ser considerado como estrategias de mejora, las cuales serán aportadas por el proyecto de modernización a la planta.

La tabla 3, muestra el mapa logrado por medio de la calificación subjetiva de la relación factor operacional-estrategia de mejora, en el proyecto de modernización de este caso de estudio. Al asignar una escala de calificación entre -2 a +2 con cero al centro, similar a la usada para valorar la matriz de valoración del vector VCEE, y usando el criterio de que las dificultades se evalúan con escala inversa respecto a los beneficios ${ }^{4}$, se obtuvo una medida indirecta del Vector de Cambio de los Efectos Internos (VCEI). 
Tabla 3. Matriz de valoración del Vector de Cambios en los Efectos Internos (VCEI)

\begin{tabular}{|c|c|c|c|c|c|c|c|}
\hline $\begin{array}{c}\text { Factor } \\
\text { Operacional } \\
\downarrow\end{array}$ & $\begin{array}{l}\text { Mejora y } \\
\text { rapidez en las } \\
\text { decisiones } \\
\text { locales }\end{array}$ & $\begin{array}{l}\text { Reducción de } \\
\text { tiempos } \\
\text { muertos de los } \\
\text { materiales en el } \\
\text { proceso }\end{array}$ & $\begin{array}{l}\text { Meioras en el } \\
\text { flujo de } \\
\text { información } \\
\text { local }\end{array}$ & $\begin{array}{l}\text { Mejoras en el } \\
\text { flujo de } \\
\text { información al } \\
\text { exterior }\end{array}$ & $\begin{array}{l}\text { Remover los } \\
\text { cuellos de } \\
\text { botella internos, } \\
\text { materiales e } \\
\text { información }\end{array}$ & $\begin{array}{l}\text { Remover los } \\
\text { cuellos de } \\
\text { botella externos }\end{array}$ & $\begin{array}{c}\text { Mejora en la } \\
\text { sccuencia y } \\
\text { operación del } \\
\text { proceso }\end{array}$ \\
\hline & $E_{1}$ & $\mathrm{E}_{2}$ & $\mathrm{E}_{3}$ & $\mathrm{E}_{4}$ & $\mathrm{E}_{5}$ & $\mathrm{E}_{6}$ & $\mathrm{E}_{7}$ \\
\hline Dificultad tecnológica & $\begin{array}{c}\text { Baja } \\
+2\end{array}$ & $\begin{array}{c}\text { Baja } \\
+2\end{array}$ & $\begin{array}{c}\text { Baja } \\
+2\end{array}$ & $\begin{array}{c}\text { Baja } \\
+2\end{array}$ & $\begin{array}{c}\text { Media } \\
0\end{array}$ & $\begin{array}{c}\text { Media } \\
0\end{array}$ & $\begin{array}{c}\text { Baja } \\
+2\end{array}$ \\
\hline Dificultad organizacional & $\begin{array}{c}\text { Alta } \\
-2\end{array}$ & $\underset{0}{\text { Media }}$ & $\underset{0}{\text { Media }}$ & $\begin{array}{c}\text { Alta } \\
-2\end{array}$ & $\begin{array}{c}\text { Alta } \\
-2\end{array}$ & $\begin{array}{c}\text { Alta } \\
-2\end{array}$ & $\begin{array}{c}\text { Media } \\
0\end{array}$ \\
\hline Dificultad por actitudes & $\begin{array}{c}\text { Alta } \\
-2\end{array}$ & $\begin{array}{c}\text { Media } \\
0\end{array}$ & $\begin{array}{c}\text { Media } \\
0\end{array}$ & $\begin{array}{c}\text { Alta } \\
-2\end{array}$ & $\begin{array}{c}\text { Alta } \\
-2\end{array}$ & $\begin{array}{c}\text { Alta } \\
-2\end{array}$ & $\begin{array}{c}\text { Media } \\
0\end{array}$ \\
\hline $\begin{array}{l}\text { Beneficio de la planta } \\
\text { individual }\end{array}$ & $\begin{array}{c}\text { Medio } \\
0\end{array}$ & $\begin{array}{c}\text { Medio } \\
0\end{array}$ & $\begin{array}{c}\text { Medio } \\
0\end{array}$ & $\begin{array}{l}\text { Alto } \\
+2\end{array}$ & $\begin{array}{c}\text { Alto } \\
+2\end{array}$ & $\begin{array}{c}\text { Alto } \\
+2\end{array}$ & $\begin{array}{c}\text { Alto } \\
+2\end{array}$ \\
\hline $\begin{array}{l}\text { Sistema de beneficios } \\
\text { colectivoso o en cadena } \\
\text { derivados del proyecto }\end{array}$ & $\begin{array}{c}\text { Bajo } \\
-2\end{array}$ & $\begin{array}{c}\text { Bajo } \\
-2\end{array}$ & $\begin{array}{c}\text { Medio } \\
0\end{array}$ & $\begin{array}{l}\text { Alto } \\
+2\end{array}$ & $\begin{array}{c}\text { Bajo } \\
-2\end{array}$ & $\begin{array}{l}\text { Alto } \\
+2\end{array}$ & $\begin{array}{l}\text { Alto } \\
+2\end{array}$ \\
\hline Valor de las estrategias & $E_{1}-4$ & $\mathrm{E}_{2}=0$ & $\mathrm{E}_{3}=+2$ & $\mathrm{E}_{4}=+2$ & $E_{5}=-4$ & $E_{6}=0$ & $E_{7}=+6$ \\
\hline
\end{tabular}

En la matriz resalta lo siguiente:

* El proyecto de modernización representa baja dificultad tecnológica en su implementación.

* Presenta dificultades con calificación de medio a alta en su implementación, debido a factores de actitud y organizacionales.

* Significa un alto beneficio individual y colec. tivo para mejorar los flujos de información al exterior, remover los cuellos de botella externos e internos en el flujo de materiales e información y mejora en la secuencia de operación del proceso.

* El Vector de Cambios en los Efectos Internos resulta de un valor positivo y marginal, dado que:

$$
\sum_{i=1}^{7} E_{i}=+2
$$

\section{Evaluación económica-financiera}

La tercer vertiente del proceso de evaluación, la conforma la evaluación económica tradicional que es realizada a partir de la determinación de los costos asociados con el proyecto y su relación con los beneficios, el tiempo y la tasa de retorno de la inversión, entre muchos otros indicadores de índole financiero y económico.

Cabe destacar, que para aplicar las fórmulas financieras básicas para obtener los indicadores, se requirió hacer una traducción de los beneficios a términos económicos, y previo a esto, hacer la determinación de cuáles serían las áreas de la planta, que una vez ejecutado el proyecto de modernización, podrían aportar beneficios.
Determinación de los costos

En el proyecto de ingeniería, se determinaron los costos globales asociados con la propuesta de ingeniería básica para la modernización de la instrumentación y automatización de la planta Chapultepec. En esta fase, se obtuvieron los costos de todos y cada uno de los nuevos instrumentos y sistemas de control propuestos, así como de los costos de arranque y refaccionamiento ${ }^{5}$.

\section{Determinación de áreas de beneficio}

El siguiente paso, fue la determinación de las áreas que pueden presentar beneficios potenciales con los cambios propuestos.

Para tal efecto, se eligió y propuso como metodología el uso de algunas herramientas de evaluación tecnológica adaptadas y modificadas de tal forma que fueran aplicables a nuestros fines de evaluación.

Invirtiendo los conceptos usados en la matriz de portafolio de riesgos, utilizada por Taggar y Blaxter (1992), en lugar de hacerla una matriz con elementos de riesgo, en los elementos básicos individuales de la matriz, se conceptualizó como una matriz cuyos elementos son las áreas de oportunidad, comparadas o mapeadas contra los elementos de beneficio.

\footnotetext{
${ }^{4}$ Esto quiere decir que un factor que presenta baja dificultad tiene una calificación positiva de +2 , mientras que uno de alta dificultad tiene una calificación de -2 , por lo tanto, los altos beneficios se califican con +2 y los bajos beneficios de -2 .

${ }^{5}$ Estos resultados no se muestran, ya que están fuera del alcance de este trabajo, y forman parte del proyecto de ingeniería básica.
} 
Con el procedimiento anterior, es posible identificar aquellas casillas de la matriz, cuyo desarrollo e implementación permitieran justificar el proyecto desde el punto de vista económico, así como darle componentes de viabilidad financiera.

Es importante aclarar que no se contempla como criterio de beneficio del proyecto, el reducir costos de mano de obra por despedir a los operadores si se moderniza la automatización y control. Este criterio es congruente con los atributos de satisfacción expuestos en la tabla 1, por otra parte, si el proyecto se aceptara y fuera ejecutado, se podrán mejorar y facilitar las tareas del personal, además de su nivel técnico al capacitarse para asimilar las nuevas tecnologías disponibles.

Esquemáticamente, la operación de la planta es sencilla, según se muestra en la siguiente figura:

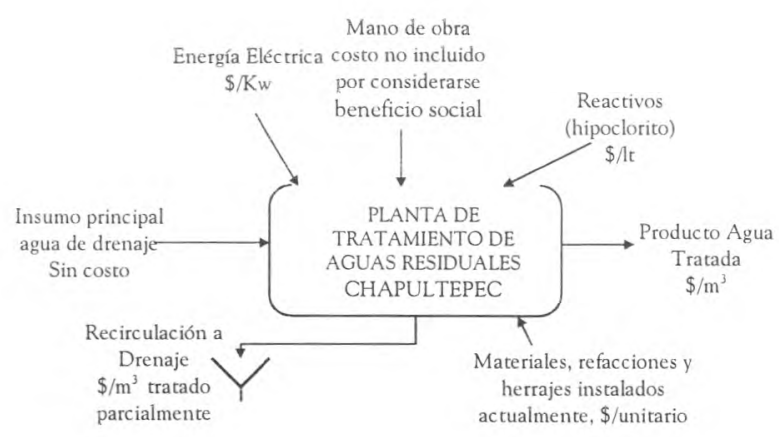

\section{Figura 4. Enfoque sistémico de entradas-salidas a la PTAR Chapultepec}

El criterio utilizado para la construcción de las columnas de la matriz de que con la consigna de mantener o aumentar la eficiencia de la planta, se considera beneficio relativo a la ejecución de la modernización propuesta a:

* Todo el ahorro de los insumos que tienen un costo asociado; por ejemplo, la energía o los reactivos utilizados.

* Todo aquel subsistema, área, dispositivo o instrumento que contribuya con el aumento en la productividad.

* Todo subsistema, área, dispositivo o instrumento, que contribuya con el aumento en la calidad del producto.

* Todo aquel subsistema, área, dispositivo o instrumento que contribuya con el aumento en la seguridad de la planta.

* Todo aquel subsistema, área, dispositivo o instrumento que contribuya con la disminución de desperdicios de cualquier índole.

* Todo aquel subsistema, área, dispositivo o instrumento que contribuya con el aumento o mejora en la manejabilidad, explotación de la información y administración de la planta en su conjunto.

En los renglones, consideramos como áreas de estudio de la planta aquellas identificadas en la descripción de los procesos unitarios de los diagramas de tuberías e instrumentación, o las líneas de enlace entre operaciones unitarias con la instrumentación respectiva propuesta en el proyecto de modernización.

La detección de las áreas de oportunidad se basó en los siguientes juicios:

1. Toda casilla en donde se marca como alto el beneficio de alguna columna, señaló el área de la planta por revisar y estudiar cuidadosamente, para determinar en qué consistían los beneficios.

2. Cualquier área de la planta en donde las casillas marquen más de un beneficio alto o medio, también deberá ser objeto de revisión.

3. Cualquier beneficio, (columna que califique con al menos tres medios en diferentes áreas), será considerado como de alto provecho, por lo tanto, su impacto deberá evaluarse.

En el caso de la unidad evaluada en la tabla 4 se muestra específicamente la siguiente posibilidad de áreas (renglones):

* Son posibles altos ahorros de energía en los turbo sopladores.

* El aumento en la calidad del producto puede obtenerse en la operación combinada del tanque de aireación TA-11 y de los turbo sopladores, ya que la calidad del agua tratada depende de que exista la concentración adecuada de oxígeno disuelto en el tanque de aireación.

* Es posible obtener un alto ahorro de reactivos en el tanque de hipoclorito TA-15.

* El hecho de utilizar una consola de control central, podría incidir en un alto aumento a la productividad, en mejoras a la administración de la planta y en la seguridad de la misma.

Por beneficios (columnas):

* Ahorro de energía en los turbo sopladores.

* Ahorro de reactivos en el tanque de cloración y su circuito de control.

* Aumento de calidad en el producto, en la operación del tanque de aireación-turbo sopladores y en el tanque sedimentador secundario (Turbiedad).

* Ahorro de desperdicios en: tanque sedimentador primario, tanque sedimentador secundario, (recirculaciones y exceso al drenaje), así como en el tanque de cloración.

* Aumento en la cantidad del producto: tanques sedimentadores, tanque de aireación. 
Tabla 4. Matriz para la determinación de áreas de oportunidad de beneficio

\begin{tabular}{|c|c|c|c|c|c|c|c|}
\hline Beneficios $\rightarrow$ & $\begin{array}{l}\text { Ahorro de } \\
\text { energía }\end{array}$ & $\begin{array}{l}\text { Ahorro de } \\
\text { reactivos }\end{array}$ & $\begin{array}{l}\text { Aumento en la } \\
\text { productividad }\end{array}$ & $\begin{array}{l}\text { Aumento en la } \\
\text { calidad del } \\
\text { producto }\end{array}$ & $\begin{array}{l}\text { Disminución de } \\
\text { desperdicios }\end{array}$ & $\begin{array}{l}\text { Mejora en la } \\
\text { administración } \\
\text { de la planta }\end{array}$ & $\begin{array}{l}\text { Aumento en la } \\
\text { seguridad }\end{array}$ \\
\hline \multicolumn{8}{|l|}{$\begin{array}{l}\text { Área de la planta } \\
\text { modernizada }\end{array}$} \\
\hline 1 Entrada del influente & & & & & & Bajo & \\
\hline $\begin{array}{l}2 \text { Desengrasador y tanque } \\
\text { sedimentador primario } \\
\text { TA-09 y TA-10 }\end{array}$ & & & & Bajo & Bajo & Bajo & \\
\hline $\begin{array}{l}3 \text { Tanque de aireación } \\
\text { TA-11 }\end{array}$ & & & & Alto & \multicolumn{3}{|c|}{ Medio } \\
\hline 4 Turbosopladores & Alto & & \multicolumn{3}{|c|}{ Alto } & \multicolumn{2}{|l|}{ Medio } \\
\hline $\begin{array}{l}5 \text { Línea de turbo } \\
\text { sopladores a tanques de } \\
\text { aireación }\end{array}$ & & & & & & \multirow[t]{2}{*}{ Bajo } & \\
\hline $\begin{array}{l}6 \text { Línea entre el tanque de } \\
\text { aireación y el tanque } \\
\text { sedimentador } \\
\text { secundario }\end{array}$ & & & & & & & \\
\hline $\begin{array}{l}7 \text { Tanque sedimentador } \\
\text { secundario TA-12 }\end{array}$ & & & & \multirow[t]{2}{*}{ Medio } & Medio & Medio & \\
\hline $\begin{array}{l}8 \text { Tanque de cloración } \\
\text { TA-15 }\end{array}$ & \multicolumn{3}{|c|}{ Alto } & & Medio & Bajo & Bajo \\
\hline $\begin{array}{l}9 \text { Línea del tanque de } \\
\text { hipoclorito GA-01 al } \\
\text { tanque de cloración } \\
\text { TA-15 y TA-05 de la } \\
\text { unidad } 2\end{array}$ & & & & & & Bajo & \\
\hline $\begin{array}{l}10 \text { Consola de control } \\
\text { central }\end{array}$ & Bajo & Bajo & Alto & Medio & Medio & Alto & Alto \\
\hline
\end{tabular}

* Mejora en la administración de la planta, prácticamente en todas las áreas.

\section{Análisis de los Beneficios ${ }^{6}$}

Se utilizaron como métodos de análisis financiero, el Valor Presente Neto (VPN) y la relación Costo/Beneficio. El primero de ellos, plantea la visión de que la inversión requerida para el proyecto, pudiera ser realizada por una empresa privada, mientras que el segundo se basa en la visión complementaria de que la planta pertenece a una dependencia gubernamental. Para este caso, Newman (1996), señaló que:

"el análisis económico gubernamental es el método de la relación beneficio/costo, debido a la legislación federal... y se ha convertido en un método tan familiar para la gente del gobierno como lo es la tasa de rendimiento para la gente de la industria".

Para el desarrollo, se requirió el cálculo del flujo de efectivo que es la diferencia entre el total de efectivo que se recibe (ingresos o beneficios) y el total de erogaciones (egresos o inversiones) para un período anual.

El medio más fácil para imaginarse el flujo de efectivo es por medio del diagrama del mismo nombre, en el que cada flujo individual se representa como una flecha a lo largo de

${ }^{6}$ Las cantidades, precios y tipo de cambio peso/dólar EUA mostrados y usados en las secciones de análisis de costos financieros están referidas al año de 1997. la escala de tiempo horizontal, los flujos positivos se representan como flechas hacia arriba y los flujos negativos apuntan hacia abajo. Se supone que cada flujo ocurre al final del período respectivo.

Durante la vida útil de la planta de tratamiento, se tienen costos de producción, los que se subdividen en costos de operación y mantenimiento.

Una inversión es el gasto que en se incurre para la adquisición o instalación de un bien duradero, mientras que un costo de operación se asocia con los bienes y servicios que se consumen directamente durante el proceso de tratamiento del agua residual.

El primer insumo básico para nuestro análisis es el conocimiento del valor comercial del $\mathrm{m}^{3}$ de agua tratada. La $\mathrm{DGCOH}^{7}$ nos proporcionó la siguiente información que apareció en el Diario Oficial de la Federación:

a) Precio del $\mathrm{m}^{3}$ de AT proveniente de tomas de tipo cuello de garza $\$ 6.42 \mathrm{MN}$.

b) Cuando se surte en camiones tipo cisterna para su comercialización incluyendo el transporte en el DF el 60\% de la cuota prevista en el inciso B9 de la fracción 1 del artículo 197 del Código Financiero del DDF, 31 de Diciembre de 1996; precio del $\mathrm{m}^{3}$ de AT $\$ 17.85 \mathrm{MN}$.

\footnotetext{
${ }^{7}$ Información proporcionada por funcionarios de la $\mathrm{DGCOH}$, las cantidades están en pesos mexicanos, los datos refieren al año de ejecución.
} 


\section{DOI: http://dx.doi.org/10.22201/fi.25940732e.2001.02n2.008}

\section{Ahorro de energía}

Dentro de las limitaciones impuestas por los alcances de este proyecto, se hizo evidente qùe el área en donde se concentra este ahorro se nota en el consumo mensual de energía para los turbo sopladores y fue determinada en la sección anterior.

La propuesta de mejora fue incluir un sistema para optimizar el funcionamiento de los turbo sopladores, en función de los requerimientos de oxigeno disuelto, de acuerdo con la información de diversos fabricantes ${ }^{8}$. Esto señaló la posibilidad de alcanzar ahorros de entre un 20\% a $40 \%$ del consumo permanente de energía.

Considerando el ahorro potencial mensual y anual con la implantación del nuevo sistema de control propuesto en este proyecto de modernización, se obtuvo:

De los datos de placa, la potencia del equipo es de 200 HP, transformando unidades:

$$
200 \mathrm{HP} \text { 's } * 756 \text { watts/HP }=149200 \text { watts }
$$

Costo por $\mathrm{KW} / \mathrm{Hr}$ para plantas de tratamiento, según el Diario Oficial de la Federación $\$ 0.37$ por

\section{$\mathrm{KW} / \mathrm{Hr}$,}

Por lo que el costo del consumo mensual estimado es:

$149.2 \mathrm{Kw} / \mathrm{Hr} * 0.37 \$ / \mathrm{Kw} / \mathrm{hr} * 24 \mathrm{hrs} /$ día

$* 30$ días $/$ mes $=\$ 39,747.88$

Ahorro estimado mensual

$$
30 \%=\$ 39,746.88 * 0.3=\$ 11,924.00
$$

Ahorro de energía estimado anual

$$
\$ 11,924.00 * 12=\$ 143,088.00
$$

\section{Ahorro de Reactivos}

De acuerdo con la determinación de áreas de beneficio, el único ahorro considerado de reactivos es a nivel del tanque de cloración, específicamente en el consumo mensual de hipoclorito; sin embargo, dado que los consumos y el precio del reactivo son reducidos, en realidad el ahorro potencial, al introducir el sistema de control es despreciable, si se considera como un beneficio económico, aunque por otra parte, puede mejorar la operación de la función de cloración en la planta.

\footnotetext{
${ }^{8}$ Allen Bradley, Capital Controls y Siemens, entre otros.
}

Mejoras en la productividad por la administración del proceso (Management)

La mejor gestión del proceso y su información, tanto en el intercambio como en la explotación de la misma, puede verse como un beneficio derivado del ahorro que se tiene al mejorar en el flujo de información, ya sea al interior o al exterior de la planta, así como la factibilidad de realizar análisis históricos de la información y de la totalización y registro de los caudales de agua tratados.

Por otra parte, prácticamente toda la literatura de aplicación de sistemas de control concede una gran importancia a este renglón y establece que las mejoras en la eficiencia del proceso pueden ser en rangos de hasta un $20 \%$, dependiendo de las características particulares de los mismos.

Dado que no contamos con otros indicadores para la calificación del desempeño de la planta en su interior, manejaremos uno de los más conocidos y utilizados en la contabilidad de la administración estratégica: la productividad.

Stainer (1997), la define como la utilización eficiente de entradas en la producción de los bienes y servicios que el sistema tiene como salidas. Generalmente, contiene consideraciones de mano de obra utilizada, insumos, materias primas, energía y recursos diversos. Como salidas, los niveles de producción periódicos, su incremento y la medición de los desechos respectivos, así como su impacto ambiental. Por supuesto, deben considerarse características propias de la producción, como la calidad, entre otras.

Haciendo una simplificación de todos los factores mencionados, se considerará solamente que por concepto de administración del proceso con el nuevo sistema de control propuesto en el proyecto de modernización, el incremento en la productividad será del $8 \%$ en términos de la producción mensual.

Por otra parte, si el promedio de producción de la planta es de $120 \mathrm{lts} / \mathrm{seg}$, esto representa $10,368 \mathrm{~m}^{3}$ por día o $311,040 \mathrm{~m}^{3}$ por mes.

El aumento estimado mensual será entonces de $(0.08)(311,040)=24,883.2 \mathrm{~m}^{3}$ por mes o de $298,598 \mathrm{~m}^{3}$ por año. En términos económicos, si usamos el precio unitario del metro cúbico de agua tratada inciso (a) punto de los análisis de beneficio, tenemos:

$\left(298,598 \mathrm{~m}^{3}\right.$ por año $)\left(\$ 5.00 / \mathrm{m}^{3}\right)=\$ 1,492,992.00 /$ por aก̃o ${ }^{9}$.

9 Se estima que el precio del $\mathrm{m}^{3}$ de agua tratada deberá venderse a $\$ 5.00 \mathrm{MN}$ precio inferior al comercial. 
Totalización de beneficios anuales

De los resultados del ahorro de energía, de reactivos y mejoras en la productividad para la administración del proceso, tenemos que:

$$
\underset{i=1}{B_{T}^{3}}=\sum B_{i}=\$ 143,088.00+0.0+\$ 1,492,992.00=
$$

$$
\$ 1,636,080.00 \text { anual. }
$$

Análisis de los costos

Inversión requerida y costos anuales derivados del proyecto de modernización:

\section{Inversión requerida}

De acuerdo con los resultados arrojados por el proyecto de ingeniería básica, el costo total por el proyecto de modernización es de:

\begin{tabular}{cll}
\hline \hline & \multicolumn{1}{c}{ DESCRIPCIÓN } & PRECIO Dls. EUA \\
\hline \hline I & $\begin{array}{l}\text { Equipo electrónico para el } \\
\text { sistema de control, estaciones } \\
\text { de proceso y software de } \\
\text { operación y configuración }\end{array}$ & $\$ 138,638.00$ \\
II & Equipo de campo & $\$ 157,007.00$ \\
III & Capacitación & $\$ 5,155.00$ \\
IV & Ingeniería & $\$ 11,700.00$ \\
V & Servicio & $\$ 11,263.00$ \\
VI & Lote de refracciones & $\$ 11,559.00$ \\
& TOTAL & $\$ 400,000.00$ \\
\hline \hline
\end{tabular}

Costo de operación y material para mantenimiento de adquisición local

La instalación de la nueva instrumentación, requerirá del nuevo personal para operación y mantenimiento de la misma:

Se estima el trabajo en dos turnos, por cada turno:

Sueldo de 1 supervisor de instrumentación $\$ 3,500.00$ por mes

Sueldo de 1 técnico instrumentista $\$ 2,400.00$ por mes

Total de sueldos por turno $\$ 5,900.00$ por mes

Total de sueldos por dos turnos $\$ 11,800.00$ por mes

Total 1 de sueldos anuales: $\$ 153,400.00$, incluyendo un mes de aguinaldo.

Costo de reactivos y materiales diversos necesarios en la operación diaria:

(Circuitos y componentes eléctricos y electrónicos, soldadura, empaques, sellos, baleros, papelería, etc.), cantidad fija alzada de $\$ 15,000.00$ por mes.
Total 2 de materiales diversos: $\$ 180,000.00$ por año.

Lote de refacciones solicitado cada dos años para mantenimiento

Para la correcta operación y mantenimiento de los nuevos equipos y sistemas de medición y control, la experiencia en el campo nos indica que es necesario solicitar cada dos años un lote de refacciones, así como de consumibles y reactivos, debido a la caducidad de los mismos y a los tiempos de entrega, ya que se trata de equipos de importación. Se trata fundamentalmente de piezas de desgaste, electrodos, circuitos eléctricos y electrónicos a nivel tarjeta, normalmente se requiere invertir un monto aproximado de $5 \%$ del costo de la instrumentación.

Costo del lote de refacciones bianual $(\$ 150,000.00) * 0.05$ $=\$ 7,500.00$ dólares EUA, al tipo de cambio actual $\$ 7,500.00$ $* 8=\$ 60,00.00$.

Total 3 lote de refacciones y consumibles bianual $\$ 60,000.00$.

\section{Consumo Energético ${ }^{10}$}

La nueva instrumentación requerirá de ser alimentada eléctricamente, según la siguiente estimación:

Consumo aproximado del sistema de control central y variador de velocidad 10 amperes.

Consumo aproximado del equipo de campo: 50 transmisores por $20 \mathrm{mACD} / \mathrm{cada}$ uno $=1 \mathrm{Ampere}$, Fuentes de CD, Analizadores aprox. $7 \mathrm{Amps}$, por lo que tenemos un total aproximado de $20 \mathrm{~A}$ consumidos por todo el sistema.

Potencia total consumida:

Total $20 \mathrm{~A} * 120 \mathrm{VCA}=48 \mathrm{KW} * \$ 0.37 / \mathrm{KW} / \mathrm{Hr}$ $* 24 * 30=\$ 12,787.00$ por mes.

Total 4 consumo energético anual $\$ 12,787.00 * 12=$ $\$ 153,446.00$.

\section{Evaluación económico-financiera}

Con la información económico-financiera desarrollada y obtenida en los puntos anteriores, podemos trabajar para la obtención de índices, la relación beneficio/costo o el valor presente neto. Dado que se trabaja con los datos del mismo diagrama de flujos de efectivo, los resultados en cuanto a los criterios de aceptabilidad del proyecto son equivalentes.

\footnotetext{
${ }^{10}$ Nota: cl aire de instrumentos se toma de la planta, por lo tanto, su costo es despreciable para los fines de este estudio.
} 


\section{Valor Presente Neto (VPN)}

Este método consiste en determinar la equivalencia en el tiempo cero de los flujos futuros de efectivo que genera el proyecto y comparar esta equivalencia con las inversiones hechas. Cuando la equivalencia es positiva, es decir, VPN $>0$ el proyecto se acepta.

Fórmula utilizada para evaluar el valor actualizado neto de los flujos generados en el proyecto:

Donde:

$$
\operatorname{VPN}=\Sigma(B k-E k)(1+i)-k \quad n
$$

VPN $=$ Valor Presente Neto

$\mathrm{i}=$ Tasa de interés anual, considerada en por ciento.

$\mathrm{n}=$ Número de años de la vida útil del proyecto

$\mathrm{Bk}=$ Beneficio en el período $\mathrm{k}$ - ésimo

Ek = Inversión en el período k-ésimo-

En la fórmula se considera el valor del dinero a través del tiempo al seleccionar un valor adecuado de y. Para los análisis, se considera una tasa de interés anual y tanto los beneficios como las inversiones están en base anual.

Los criterios de aceptación del proyecto son:

$\cdot V P N>0 \quad$ Se acepta el proyecto

$\cdot V P N=0 \quad$ Es indiferente

VPV $<0$ Se rechaza.

Para nuestro proyecto de modernización, considerando $\mathrm{n}=20, \mathrm{i}=25 \%$, los cálculos respectivos son los siguientes:

$$
\begin{gathered}
\mathrm{VPN}=(0-3,200,000.00) \\
+[(1,636,078.00-486,846)][1.25-1+1.25-3+1.25-5 \\
+1.25-7+1.25-9+1.25-11+1.25-13+1.25-15 \\
\cdot \quad+1.25-17+1.25-19 *]+ \\
+[(1,636,078-546,846)][1.25-2+1.25-4+1.25-6 \\
+1.25-8+1.25-10+1.25-12+1.25-14+1.25-16 \\
+1.25-18+1.25-20]=1,266,461.00>0
\end{gathered}
$$

El indicador financiero es positivo, por lo que el proyecto es aceptado.

\section{Relación beneficio/costo}

Este indicador es resultado de dividir el valor actualizado neto de los beneficios, entre el valor actualizado neto de las inversiones. La actualización se realiza basándose en una tasa de interés dada, generalmente conocida como TREMA o tasa de recuperación mínima atractiva. Se expresa como:

$$
\frac{B}{C}=\frac{\sum_{k=1}^{n} B_{k}(1+i)^{-k}}{\sum_{k=1}^{n} E_{k}(1+i)^{-k}}
$$

Donde

$$
\begin{aligned}
& B / C=\text { Relación Benefico/Costo } \\
& I=\text { Tasa de interés anual considerada en por ciento } \\
& \mathrm{N}=\text { Número de años de vida útil del proyecto } \\
& \mathrm{B}_{\mathrm{k}}=\text { Beneficio en el período } \mathrm{k} \\
& \mathrm{E}_{\mathrm{k}}=\text { Inversión en el período } \mathrm{k}
\end{aligned}
$$

Los criterios de aceptación del proyecto son:

$\begin{array}{ll}B / C>1 & \text { Se acepta } \\ B / C=1 & \text { Es indiferente } \\ B / C<1 & \text { Se rechaza }\end{array}$

En nuestro caso, con $\mathrm{n}=20$ y con $\mathrm{i}=25 \%$ anual, tenemos la ecuación 1.

Por lo que utilizando este indicador financiero, el proyecto también es aceptable.

\section{Evaluación final integrada}

El análisis de factibilidad para el proyecto de modernización de este estudio fue calificado como positivo en su evaluación integral, ya que:

Vector de Cambios en los Efectos Externos

VCEE $=+83$ Puntos (Positivo)

Vector de Cambios en los Efectos Internos $\mathrm{VCEI}=+2$ Puntos (positivo marginal)

Ecuación 1

$$
\begin{gathered}
\frac{B}{C}=\frac{(1,636,078)\left[1.25^{-1}\right]+1.25^{-2}+1.25^{-3}+1.25^{-4}+\ldots+1.25^{-20}}{(3,200,000)\left(1.25^{0}\right)+[486,846]\left[1.25^{-1}+1.25^{-3}+\ldots+1.25^{-19}\right]+[546,846]\left[1.25^{-2}+1.25^{-4} \ldots+1.25^{-20}\right]} \\
\frac{B}{C}=\frac{6,508,612.77}{6,041,819.95}=1.077>1
\end{gathered}
$$


Valoración Financiera del Valor Presente Neto

VPN $>0$, o Relación B/C>1

Lo cual corresponde al último renglón de la tabla 1 de Criterios de Aceptación de Proyectos mostrada anteriormente.

\section{Conclusiones}

La metodología propuesta de evaluación integral de los vectores de cambios externos, internos y la evaluación económico-financiera básica, resulta exitosa para la toma de decisiones y la aceptación o rechazo de proyectos de modernización.

Un caso particular de esta metodología es la evaluación de nuevos proyectos de inversión. En esta situación el Vector de Cambios en los Efectos Internos VCEI es cero por lo que aún no existe una organización, ni instalaciones previas.

Para la sección de análisis financiero, hemos supuesto que en una planta de tratamiento de aguas residuales operada gubernamentalmente, el proyecto de modernización generará un aumento en la productividad de agua tratada comercializable. Este sería el caso de una unidad productiva de tratamiento de aguas privada; ya que de otra forma, el cálculo financiero pierde sentido.

En el cálculo de los índices financieros, se consideró el precio del $\mathrm{m}^{3}$ de agua tratada en $\$ 5.00 \mathrm{MN}$, inferior al señalado de $\$ 6.42 \mathrm{MN}$ en tomas cuello de garza, pensando que la venta será realizada a pipas o estaciones de servicio automotriz, entre otras posibilidades. Los indicadores resultaron favorables aún con el descuento en el precio.

Para los cálculos, se estimó un aumento en la productividad de un mínimo de $8 \%$ aunque los reportes y literatura comercial sobre proyectos de modernización en la instrumentación y control de plantas de proceso diversas, señalan que la productividad puede aumentar entre un 20 a un $30 \%$ debido a la mejor administración del proceso.

Por otra parte, la evaluación social de proyectos, frecuentemente se asocia con la toma de decisiones para optimizar recursos, dentro de un problema de selección de proyectos que tengan un mayor impacto sobre el contexto de la economía de un país. En el caso analizado para este informe metodológico, se ha demostrado que la evaluación de impacto social puede realizarse a un

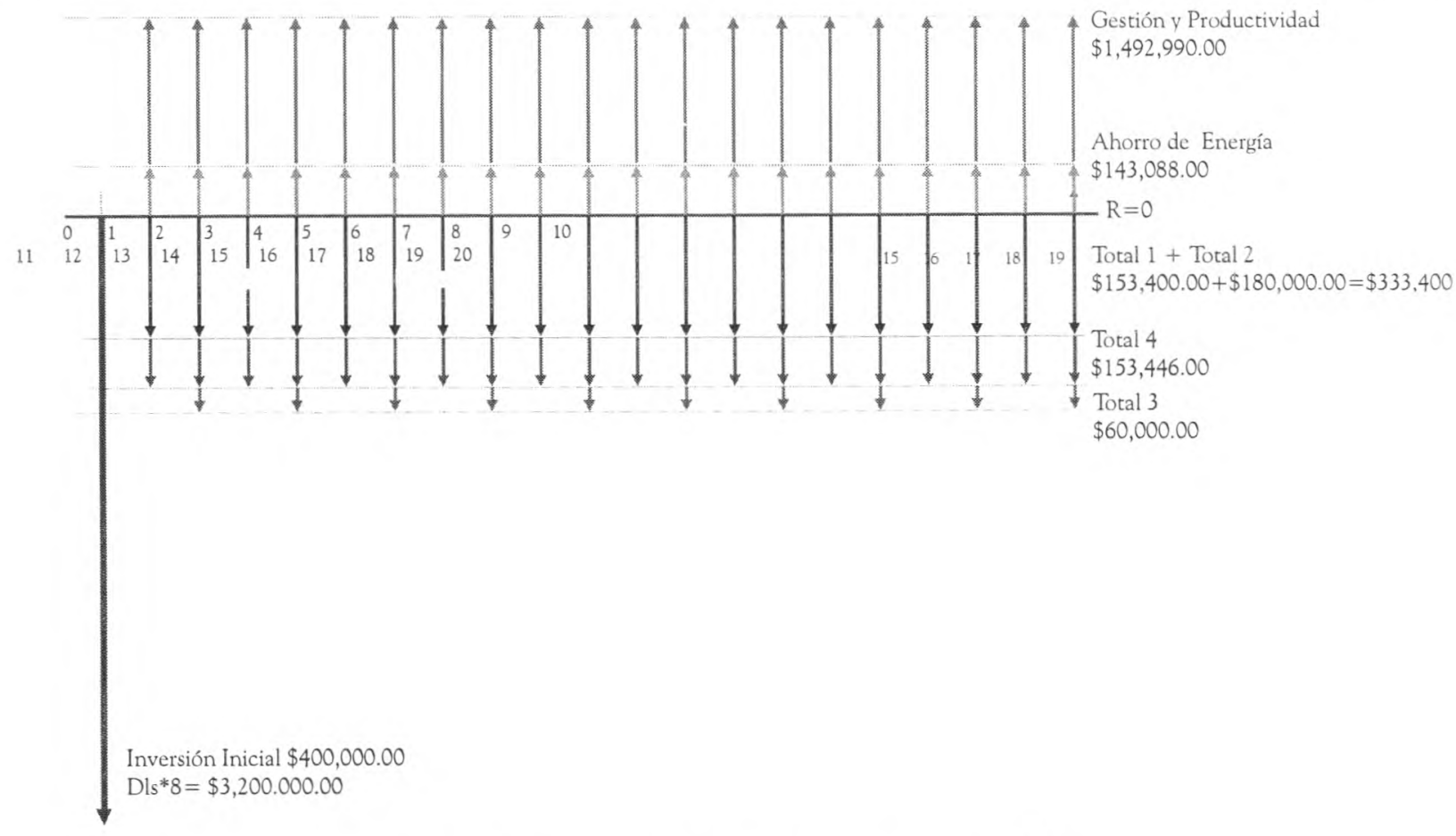

Figura 5. Diagrama de Flujos de Efectivo (DFE)

Consideraciones para la construcción del DFE

Se mantienen tanto los costos como los beneficios a precios fijos a valor presente para hacerlos comparables.

El valor de recuperación de los equipos al término de su vida útil es despreciable.

La vida útil del equipo en promedio se estimó en 20 años, si se mantiene en forma preventiva y correctiva oportunamente.

El tipo de cambio considerado para los cálculos se refiere al año de realización de este proyecto. 
proyecto aislado de modernización, al interior de una unidad productiva y aportar elementos de decisión para la aceptación o rechazo del proyecto.

\section{Agradecimientos}

Agradezco a la empresa INCAM su autorización para la publicación de los datos e información técnica sobre la metodología propuesta y usada para la evaluación del proyecto de modernización de la instrumentación y control de la PTAR Chapultepec, así como a la Dirección Técnica de la Dirección General de Construcción y Operación Hidráulica del Gobierno del Distrito Federal mexicano por su autorización para la publicación de algunos datos técnicos de la mencionada planta.

\section{Referencias}

Gitman L.J. (1995). Fundamentos de administración financiera. Oxford University Press, Edición Harla, México.

Hinterhuber H.H. (1997). Strategic Cost Management: Preliminary Lessons from European Companies, International Journal of Technology Management, Special Issue on Strategic Cost Management, Vol.13, No.1, $1-14$.

Nardini A. (1997). A Proposal for Integrating Environmental, Impact Assesment, Cost-Benefit Analysis and Multicriteria Analysis in Decision Making, Project
Appraisal. Beech Tree Publishing, Vol.12, No.3, 173-184.

Ranashinge M. (1997). Reconciling Private Profitability and Social Costs: the Case of Clay Mining in Sri Lanka, Project Appraisal. Beech Tree Publishing, Vol.12, No.1, 31-43.

Ruffat J. (1993). Strategic Management of Public and Not Market Corporations. En: Strategic Planning for Public Service and non Profit Organizations, Edited by John Bryson, University of Minnesota, Long Range Planning, Pergamon, pp 51-64. USA.

Sciara A.J. (1996). Evaluación Social en la guía para la formulación y evaluación de proyectos de inversión. FONEP, Nacional Financiera, México.

Soumelis G.C. (1977). Project Evaluation Methodologies and Techniques. United Nations Educational, Scientific and Cultural Organization, UNESCO, Paris, France.

Stainer A. (1997). Productivity and Strategic Management Accounting. Int. Journal on Technology Management, Special Issue on Strategic Cost Management, Vol. 13, No.1, 57-67.

Taggart J.H. y Blaxter T.J. (1992). Strategy in Pharmaceutical R\&D: a Portfolio Risk Matrix. R\&D Management $22,3,241-254$.

Towill D.R. (1997). The Seamless Supply Chain-The Predator's Strategic Advantage, Int. Journal of Technology Management, Special Issue on Strategic Cost Management, Vol. 13, No. 1, 37-56.

\section{Semblanza del autor}

Luis Roberto Vega-González. Egresó como ingeniero mecánico electricista de la Facultad de Ingeniería, UNAM, en el área de sistemas de instrumentación y control. Obtuvo su maestría en ingeniería de planeación por la DEPFI, UNAM, y otra más en administración de la organización en la Facultad de Contaduría y Administración de la UNAM. Cuenta con una especialización en administración de la tecnología realizada en el Centro para la Innovación Tecnológica de la UNAM; ha colaborado en diferentes empresas con proyectos en el área de sistemas de control y de potencia. Ha fungido como docente a nivel medio superior y maestría en la Facultad de Ingeniería, la Facultad de Contaduría y Administración, UNAM, el Instituto Tecnológico de Monterrey CCM y la Universidad Autónoma Metropolitana. Actualmente colabora en la Unidad de Inteligencia y Tecnología y es gerente del Departamento de Ingeniería y Servicio del Centro de Instrumentos de la UNAM. 